\title{
Compactness and chromatic number
}

by

\author{
Walter Taylor* (Boulder, Colo.)
}

This paper is a continuation of [5], in which we gave an example of an atomic-compact relational structure which is not a retract of a compact topological relational structure. That example was a graph of infinite chromatic number; in this paper we show that infinite chromatic number is necessary for such an example. That is, we generalize the notion of chromatic number and show (Theorem 3.2) that an atomic-compact relational structure is a retract of a compact topological relational structure if and only if none of its chromatic numbers is an infinite cardinal.

$\S 0$ contains the preliminaries. In $\S 1$ we develop the notion of chromatic number, and in $\S 2$ we relate this notion to the notion of pure extension. The main result is in $\S 3 . \S 4$ contains a characterization of retracts of compact topological relational structures in terms of ultrapowers.

The author is indebted to J. Mycielski for advice on the presentation of this material.

0. Preliminaries. We let $\mathfrak{A}=\left\langle A, R_{l}\right\rangle_{t \in T}$ denote a relational structure (with no operations and no constants). If $A$ is a (compact) topological space (not necessarily Hausdorff), and each $n$-ary $R_{t}$ is closed in $A^{n}$, then we say that $\mathfrak{A}$ is a (compact) topological relational structure. Consult [7] for the following notions: formula with constants in $\mathfrak{A}$, satisfiability of a set of formulas with constants in $\mathfrak{A}$, purity, homomorphism, atomic compactness, and retract. We will not distinguish between the designation of a predicate symbol and the relation to which it refers. We take $x$ and $y$ to be variables, and $a$ to be any fixed element of $A$. We take " $0=y$ " as representing equality in the formal language, whereas " $x=y$ " will mean that $x$ and $y$ are the same variable.

If $R$ is an $n$-ary relation on the set $A$, we let $\bar{R}$ denote the closure of $R$ in $(\beta A)^{n}$, where $\beta A$ denotes the Stone-Cech compactification of $A$. If $\mathfrak{A}$ is as above, then $\beta \mathfrak{Z}$ denotes the relational structure $\left\langle\beta A, \bar{R}_{t}\right\rangle_{t \in T}$. $\beta A$ will be taken as equal to the set of ultrafilters on $A$, which will be

* This work was supported by NSF Grant GP-5686. 
denoted $\lambda, \mu, v$ (possibly subscripted). For information on the topology of $\beta A$, consult e.g. [2].

By cardinal, finite or infinite, denoted $s$, we mean an initial ordinal (which we take to be the set of smaller ordinals). We let $k$ denote a finite cardinal. Also we adopt a symbol $\infty$ with the convention that $s<\infty$ for every s.

1. Chromatic numbers. Let us be given $n$, a positive integer, and $\varrho$, an equivalence relation on the set $\{1,2, \ldots, n\}$.

Definition 1.1. $S(\varrho, \aleph)$ is the relational structure $\langle\ll, S\rangle$, where $S$ is the $n$-ary relation defined as follows:

$$
\text { (*) } \quad\left\langle x_{1}, \ldots, x_{n}\right\rangle \& S \text { iff } \quad i \varrho j \Rightarrow x_{i}=x_{j} .
$$

Definition 1.2. Let $R$ be an $n$-ary relation on the set $A . \chi_{\varrho}(R)$, the $\varrho$-chromatic number of $R$, is the least $s$ such that there is a homomorphism $F:\langle A, R\rangle \rightarrow \subseteq(\varrho, \aleph)$. If no such homomorphism exists, we put $\chi_{\rho}(R)=\infty$.

To see that this definition generalizes the usual definition of chromatic number, we will suppose that $R$ is a symmetric antireflexive binary relation on $A$. Now if we take $\varrho$ such that $1 \varrho 2$, then $S_{(\varrho, N)}$ is just the complete graph on $s$ vertices. Thus the homomorphism $F$ in Definition 1.2 is a coloring of the graph $\langle A, R\rangle$ in $s$ colors. It is easy to check that our definition agrees with that of Erdös and Hajnal [1, Definition 2.8], in the case of uniform set-system.

LEMNA 1.3. Suppose there is a homomorphism $F:\langle A, R\rangle \rightarrow\langle B, S\rangle$. Then $\chi_{e}(R) \leqslant \chi_{e}(S)$.

LEMMA 1.4. Suppose $\mathfrak{B}=\langle B, T\rangle$ is a compact topological relational structure. Then $\chi_{g}(T)$ is finite or $\infty$.

Proof. Suppose that $\chi_{e}(T)$ is an infinite cardinal. We let $\Theta$ be the set of equivalence relations $\theta$ on $B$ such that the set of $\theta$-equivalence classes is finite. $\Theta$ becomes a directed set if we take $\theta_{I} \leqslant \theta_{2}$ to mean that $x \theta_{2} y \Rightarrow x \theta_{1} y$. Since $\chi_{e}(T)$ is infinite, it follows, by $(*)$, that for each $\theta \in \Theta$, we may find $\left\langle x_{10}, \ldots, x_{n \theta}\right\rangle \in T$ such that $x_{i 0} \theta x_{j \theta}$ if $i \varrho j$. Take $i$ to be one member of one $\varrho$-equivalence class, and let some subnet of $\left\langle x_{i \theta}\right\rangle$ converge to $x_{i} \in B$. Clearly each $\left\langle x_{j 0}\right\rangle$ converges to $x_{i}$ for $j e i$. Continuing this process, we clearly can find $\left\langle x_{1}, \ldots, x_{n}\right\rangle \in \bar{T}=T$, such that $i \varrho j \Rightarrow x_{i}$ $=x_{j}$. Thus $\chi_{e}(T)=\infty$. QED

CoRolLARY 1.5. Suppose $\mathfrak{A}=\langle A, R\rangle$ is a retract of a compact topological relational structure. Then $\chi_{e}(R)$ is finite or $\infty$.

CoRollari 1.6. If $\chi_{e}(R)=k<s_{0}$, then $\chi_{e}(\bar{R})=k$. If $\chi_{e}(R) \geqslant s_{0}$,
Proof. Suppose first that $\chi_{e}(R)=k$. Then there is a homomorphism $F:\langle A, R\rangle \rightarrow \mathfrak{S}(\varrho, k)$. Since $\mathfrak{S}(\varrho, k)$ is finite, hence compact, we may extend $F$ to a homomorphism $\beta F:\langle\beta A, \bar{R}\rangle \rightarrow \mathbb{S}(\varrho, k)$. Thus $\chi_{e}(\bar{R}) \leqslant k$. The reverse inequality holds by Lemma 1.3.

If $\chi_{\varrho}(R) \geqslant s_{0}$, then $\chi_{\rho}(\bar{R}) \geqslant s_{0}$, by Lemma 1.3. Thus by Lemma 1.4, $\chi_{e}(\bar{R})=\infty$. Q E D

2. Chromatic number and purity. In order to state and prove our main theorem, we need the following notion of derived relations in a relational structure.

Definition 2.1. Let $\mathfrak{A}=\left\langle A, R_{t ; t \in T}\right.$ be a relational structure. We let $\mathfrak{D} \mathfrak{A}$ (the derived relational structure) be the relational structure with carrier $A$ and containing all relations derived from relations of $\mathfrak{A}$ by permutation of variables, Cartesian products and substitution of constants of $A$. That is, the set $W$ of relations of $\mathfrak{D} \mathfrak{U}$ is the smallest set of relations, containing the relations of $\mathfrak{A}$, and subject to the following:

(D1) Let $R$ be an $n$-ary relation $\epsilon W$, and let $\pi$ be a permutation of $\{1, \ldots, n\}$. Then $W$ contains the relation $S$ defined by:

$$
\left\langle x_{1}, \ldots, x_{n}\right\rangle \in S \quad \text { iff } \quad\left\langle x_{\pi(1)}, \ldots, x_{\pi(n)}\right\rangle \in R .
$$

(D2) Let $R$ be an $n$-ary relation $\epsilon W$, and let $S$ be an $m$-ary relation $\epsilon W$. Then $W$ contains the $(n+m)$-ary relation $T$ defined by:

$\left\langle x_{1}, \ldots, x_{n}, y_{1}, \ldots, y_{n}\right\rangle \in T \quad$ iff $\quad\left\langle x_{1}, \ldots, x_{n}\right\rangle \in R \quad$ and $\left\langle y_{1}, \ldots, y_{m}\right\rangle \in S$

(D3) Let $R$ be an $(n+1)$-ary relation $\epsilon W$, and let $a \in A$. Then $W$ contains the $n$-ary relation $S$ defined by:

$$
\left\langle x_{1}, \ldots, x_{n}\right\rangle \in S \quad \text { iff }\left\langle a, x_{1}, \ldots, x_{n}\right\rangle \in R .
$$

LEMnA 2.2. If $\mathfrak{A}$ is a compact topological relational structure, then $\mathfrak{D} \mathfrak{U}$ is a compact topological relational structure under the same topology.

Lemnat 2.3. Let $R$ and $S$ be as in (D1). Then

$$
\left\langle\mu_{1}, \ldots, \mu_{n}\right\rangle \in \bar{S} \quad \text { iff } \quad\left\langle\mu_{\pi(1)}, \ldots, \mu_{\pi(n)}\right\rangle \in \bar{R} .
$$

Lamma 2.4. Let $R, S$ and $T$ be as in (D2). Then

$\left\langle\mu_{1}, \ldots, \mu_{n}, v_{1}, \ldots, v_{m}\right\rangle \in \bar{T} \quad$ iff $\left\langle\mu_{1}, \ldots, \mu_{n}\right\rangle \in \bar{R} \quad$ and $\left\langle v_{1}, \ldots, v_{m}\right\rangle \in \bar{S}$.

Lemma 2.5. Let $R$ and $S$ be as in (D3). Then

$$
\left\langle\mu_{1}, \ldots, \mu_{n}\right\rangle \in \bar{S} \quad \text { iff } \quad\left\langle a, \mu_{1}, \ldots, \mu_{n}\right\rangle \in \bar{R} .
$$

Proof. Let $U$ and $V$ be compact Hausdorff spaces, with $p: U \times V \rightarrow U$ the first co-ordinate projection, and $w$ an isolated point of $V$. One has to prove that $p[\bar{B} \cap(U \times\{w\})]=\overline{p[B \cap(U \times\{w\})]}$. It is easy to check 
the inclusion $\supseteq$. The reverse inclusion follows, since any net converging. to an element of $U \times\{w\}$ must eventually be in $U \times\{w\}$, because $U \times\{w\}$ is open. $Q \mathrm{ED}$

LEMMA 2.6. An embedding $\mathbb{F}: \mathfrak{A} \rightarrow \mathfrak{B}$ is a pure embedding iff there is a homomorphism $G: \mathfrak{B} \rightarrow \mathbb{C}$ such that $G \circ \mathbb{F}$ is an elementary embedding.

Proof. Essentially the same as ([7], Lemma 2.2).

Lemara 2.7. If $\mathfrak{A}$ is pure in $\beta \mathfrak{A}$, then $\mathfrak{D} \mathfrak{A}$ is pure in $\beta \mathfrak{D} \mathfrak{A}$.

Proof. By Lemma 2.6, there is a homomorphism $G: \beta \mathfrak{Q} \rightarrow \mathbb{C}$ such that $G \circ J$ is an elementary embedding, where $J$ is the natural inclusion of $\mathfrak{A}$ into $\beta \mathfrak{A}$. But by Lemmas $2.3-5$, the relations of $\mathfrak{D} \mathfrak{A}$ are defined by the same positive formulae as are their closures in $(\beta A)^{n}$. Thus we may apply the Theorem of Marczewski [3] to see that $G$ is a homomorphism

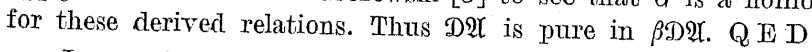

LeMna 2.8. Suppose $\mathfrak{A}$ is pure in $\beta \mathfrak{A}$, and let $R$ be an $n$-ary relation. of DIP. Then $\chi_{e}(R)$ is finite or $\infty$.

Proof. If $\chi_{e}(R)$ is an infinite cardinal, then by Cor. 1.6, $\chi_{e}(\bar{R})=\infty$. Thus there exists $\left\langle\mu_{1}, \ldots, \mu_{n}\right\rangle \in \bar{R}$, such that $i_{\varrho} j$ implies $\mu_{i}=\mu_{j}$. Since $\mathfrak{D} \mathfrak{Q}$ is pure in $\beta$ DO by Lemma 2.7 , we have $\left\langle a_{1}, \ldots, a_{n}\right\rangle \in R$, such that $i_{\varrho j}$ implies $a_{i}=a_{j}$. Thus $\chi_{e}(R)=\infty$, which is a contradiction. QE D

LEMra 2.9. Suppose $\mathfrak{I}$ is a retract of a compact topological relational structure, and $R$ is an $n$-ary relation of D2. Then $\chi_{\mathrm{e}}(R)$ is finite or $\infty$.

Proof. Similar to the above proof, using Cor. 1.5, Lemmas $2.2-5$ and 2.7 , and [3].

In the rest of this paper, when we say "every chromatic number" of a structure $\mathfrak{A}$, we mean all $\chi_{\varrho}(R)$, for every relation $R$ of $\mathfrak{A}$, and for every appropriate $\varrho$. The following theorem is the cornerstone of this paper.

THEOREM 2.10. $\mathfrak{A}$ is pure in $\beta \mathfrak{A}$ iff every chromatic number of $\mathfrak{D} \mathfrak{A}$ is finite or $\infty$

Proof. The necessity of the condition was shown in Lemma 2.8. To see sufficiency, let us assume that every chromatic number of $D \mathfrak{D}$ is finite or $\infty$. We will let $\Sigma$ be a finite set of atomic formulae of $\mathfrak{D A}$ with constants in $\mathfrak{A}$, which is satisfiable in $\beta \mathfrak{D} \mathfrak{A}$. We replace any occurrence of a constant $a$ in a relational formula by a new variable $x$, and adjoin the formula " $x=a$ ". Thus we may assume that no constant appears in a relational formula in $\Sigma$. We prove that such a $\Sigma$ is satisfiable in DR. The proof is by induction on the number of formulae in $\Sigma$.

Case I. $\Sigma$ contains no relational formulae, but only equalities. In this case there is essentially nothing to prove.

Case II. $\Sigma$ contains " $x=y$ " (where $x$ and $y$ need not be distinct). Form $\Sigma^{\prime}$ from $\Sigma$ by discarding " $x=y$ ", and replacing every occurrence
of $y$ by $x$. By induction, $\Sigma$ ' is satisfiable in $D \mathfrak{A}$, and thus so is $\Sigma$.
Case III. $\Sigma$ contains at least two relational formulae, " $R\left(x_{1}, \ldots, x_{n}\right)$ " and " $S\left(y_{1}, \ldots, y_{m}\right)$ ". Let $T$ be supplied by (D2). Form $\Sigma^{\prime}$ from $\Sigma$ by discarding these two formulae and adjoining the formula " $T\left(x_{1}, \ldots, x_{n}, y_{1}\right.$, $\left.\ldots, y_{m}\right)^{\prime \prime}$. Since $\Sigma$ is satisfiable in $\beta \mathcal{D N}$, then by Lemma $2.4, \Sigma^{\prime}$ is satisfiable in $\beta$ DOA. By induction, $\Sigma^{\prime}$ is satisfiable in DथR. Thus by (D2), $\Sigma$ is satisfiable in $\mathfrak{D} \mathfrak{A}$.

Case IV. $\Sigma$ contains " $x=a$ " and exactly one relational formula. We form $\Sigma^{\prime}$ from $\Sigma$ in the following way. First we discard " $x=a$ ". Then by (D1) we replace the given relational formula with a formula " $R(x, \ldots$, $\left.x, y_{1}, \ldots, y_{m}\right) "$, where no $y_{j}$ is $x$. Then we replace this formula with the formula "S(y $\left.y_{1}, \ldots, y_{m}\right)$ ", given by one application of (D3) for each appearance of $x$. By Lemmas 2.3 and $2.5, \Sigma^{\prime}$ is satisfiable in $\beta \mathcal{D A}$, and hence, by induction, $\Sigma^{\prime}$ is satisfiable in Dथ. Thus by (D1) and (D3), $\Sigma$ is satisfiable in $\mathfrak{D} \mathfrak{A}$.

Case V. $\Sigma$ consists of exactly one relational formula, " $R\left(x_{1}, \ldots, x_{n}\right)$ ". We define the equivalence relation $\varrho$ by taking $i \varrho j$ to mean $x_{i}=x_{j}$. Since $\Sigma$ is satisfiable in $\beta D \mathfrak{A}, \chi_{e}(\bar{R})=\infty$. Thus by Cor. 1.6, $\chi_{e}(R) \geqslant \aleph_{0}$. Thus, by hypothesis, $\chi_{e}(R)=\infty$. This means that $\Sigma$ is satisfiable in DH.

Thus we have shown that $\mathcal{D} \mathfrak{A}$ is pure in $\beta$ D) . Since $\mathfrak{A}$ is a reduct of $\mathcal{D} \mathfrak{A}$, it is clear that $\mathfrak{A}$ is pure in $\beta \mathfrak{Q}$. QED

3. The main result. The following easy theorem is a modification of a proposition of Mycielski [4, p. 4]. The main change is that we no longer require the Hausdorff axiom. This modified theorem is true even for algebras if we require that the graph of each $n$-ary operation be closed in $A^{n+1}$.

THEOREM 3.1. Every retract of a compact topological relational structure is atomic-compact.

In [5] it was shown that the converse to Theorem 3.1 is false. But we can state the following theorem relating compactness and atomic compactness.

THEOREM 3.2. The following are equivalent:

(i) $\mathfrak{A}$ is a retract of $\beta \mathfrak{X}$.

(ii) $\mathfrak{I}$ is a retract of a compact Hausdorff topological relational structure.

(iii) $\mathfrak{i}$ is a retract of a compact topological relational structure.

(iv) $\mathfrak{A}$ is atomic-compact, and every chromatic number of $\mathfrak{D} \mathfrak{A}$ is finite or $\infty$.

Proof. Obviously (i) $\Rightarrow$ (ii) $\Rightarrow$ (iii). By Lemma 2.9 and Theorem 3.1, (iii) $\Rightarrow$ (iv). Finally, (iv) $\Rightarrow$ (i) by Theorem. 2.10 and [7, Theorem 2.3]. Q E D 
The two conditions of (iv) above are independent. [5] gives an example of an atomic-compact structure with at least one number an infinite cardinal. On the chromatic that every Abelian if $\mathfrak{Y}$ is the number of number of $\mathfrak{D U}$ is finite or $\infty$, but $\mathfrak{A}$ is not atomic-compact [4, p. 2].

Suppose $\mathfrak{A}$ is a relational structure which is really an algebra, that is, each relation of $\mathfrak{A}$ is the graph of an operation. If $\mathfrak{U}$ is atomic-compact, and every chromatic number of $D \mathfrak{A}$ is finite or $\infty$, then $\mathfrak{A}$ is a retract of a compact topological relational structure. If each operation is unary, then $\mathfrak{A}$ is actually a retract of a compact topological algebra, since $\beta \mathfrak{Z}$ is an algebra. Otherwise, it is an open problem whether $\mathfrak{A}$ is a retract of a compact topological algebra.

COROLLARY 3.3. Suppose $\mathfrak{A}$ is atomic-compact and every finite reduct of $\mathfrak{U}$ is a retract of a compact topological relational structure. Then $\mathfrak{A}$ is a retract of a compact topological relational structure.

\section{Ultrapower characterization of retracts of compact topological relational} structures. The following theorem is due to Weglorz [7, Theorem 2.3]. THEOREM 4.1. A relational structure $\mathfrak{A}$ is atomic-compact iff $\mathfrak{A}$ is a re-
tract of every ultrapower of $\mathfrak{A}$.

Assuming that $\mathfrak{A}$ is actually a retract of $\beta \mathfrak{A}$, we will give a novel proof of the necessity of the condition. If $\mathfrak{A}^{I} / \mu$ is any ultrapower of $\mathfrak{A}$, then an element of $\mathfrak{U}^{I} / \mu$ is a class of functions $f: I \rightarrow A$, any two of which agree almost everywhere. Given such a function $f: I \rightarrow A$, we denote by $f(\mu)$ the ultrafilter on $A,\left\{K \subseteq A: f^{-1}[K] \epsilon \mu\right\}$. If $\mathfrak{X}$ is a retract of $\beta$, under the retraction mapping $F$, then $F(f(\mu))$ is an element of $A$. It is simple to check that $f(\mu)$ does not depend on the choice of $f$ in the It is representing an element of $\mathfrak{I}^{I} / \mu$. It is also easy to check that in this way we have constructed a retraction of the ultrapower $\mathfrak{I}^{I} / \mu$ onto r construction is an improvement on Theorem 4.1 in the following way. It is obvious that if $f(\mu)=g(\mu)$, then the class of $f$ and the class of $g$ are mapped to the same element of $A$ under this retraction. Thus we have the following definition and theorem.

DEFINITION 4.2. A consistent retraction of an ultrapower $\mathfrak{A}^{I} / \mu$ onto $\mathfrak{A}$ is a retraction $\mathbb{F}^{\mathrm{I}}$ from $\mathfrak{U}^{I} / \mu$ onto $\mathfrak{A}$, such that $f_{1}(\mu)=f_{2}(\mu)$ implies $F\left(x_{1}\right)=F\left(x_{2}\right)$, where $x_{i}$ is the class of $f_{i}(i=1,2)$

THEOREM 4.3. A relational structure $\mathfrak{A}$ is a retract of a compact topological relational structure iff $\mathfrak{U}$ is a consistent retract of every ultrapower of $\mathfrak{A}$.

Pro of. We have already found consistent retract of every ultrapower of $\mathfrak{A}$. of retracts of compact topological relational structures. To prove the
converse, we will need the following definition and lemma.
DEFINIrION 4.4 An ultrafilter $\lambda$ on the set $I$ will be called universal for the set $A$, iff given any ultrafilter $\mu$ on $A$, there is a map $f: I \rightarrow A$ such that $f(\lambda)=\mu$.

LEMMA 4.5 Let $A$ be any set. Then there is a set $I$ and an utrafilter $\lambda$ on $I$ such that $\lambda$ is universal for $A$.

Proof. Take $I$ to be the set $A^{(\beta A)}$. Then let $\lambda$ be an ultrafilter on $I$ extending the filter of subsets of the form $P_{\mu}^{-1}[K]$, where $\mu \epsilon \beta A, P_{\mu}$ is the $\mu$ th co-ordinate projection of $I$ onto $A$, and $K \in \mu$.

Returning to the proof of Theorem 4.3 , let $\lambda$ be an ultrafilter on some $I$ which is universal for the set $\bigcup_{n=1}^{\infty} A^{n}$, and let $F$ be a consistent retraction of $\mathfrak{U}^{I} / \lambda$ onto $\mathfrak{U}$. It is clear that we have a consistently defined mapping, $C$, from $\beta \mathfrak{A}$ to $\mathfrak{A}$. We need only show that $C$ is a homomorphism. Suppose $R$ is an $n$-ary relation of $\mathfrak{A}$ and we are given $\left\langle\mu_{1}, \ldots, \mu_{n}\right\rangle \in \bar{R}$. Let $\mathcal{F}$ be the filter on $A^{n}$ generated by $R$ and all products $K_{1} \times \ldots \times K_{n}$, where each $K_{i} \in \mu_{i}$. Since $\left\langle\mu_{1}, \ldots, \mu_{n}\right\rangle \in \bar{R}, \mathcal{F}$ is a proper filter, and thus we may extend $\mathcal{F}$ to an ultrafilter $y$ on $A^{n}$. Since $\lambda$ is nniversal, there is a map $f: I \rightarrow A^{n}$ such that $f(\lambda)=\nu$. Since $R \in v$, it follows that almost everywhere, $\left\langle f_{1}(i), \ldots, f_{n}(i)\right\rangle \in R$, where $f_{j}(i)$ is the $j$ th component of $f(i)$, and where $i \in I$. Thus since $F$ is a homomorphism, it follows that $\left\langle F\left(x_{1}\right)\right.$, $\left.\ldots, F\left(x_{n}\right)\right\rangle \in R$, where each $x_{j}$ is the class in $\mathfrak{A}^{I} / \mu$ with representative $f_{j}$. But since each $f_{j}(\lambda)=\mu_{j}$, it follows that $\left\langle C\left(\mu_{1}\right), \ldots, O\left(\mu_{n}\right), \in R\right.$, and thus that $C$ is a homomorphism. Q E D

\section{References}

[1] P. Erdös and A. Hajnal, On chromatic number of graphs and set-systems, Acta Math. Acad. Sci. Hungar. 17 (1966), pp. 61-99.

[2] L. Gillman and M. Jerison, Rings of continuous functions, 1960.

[3] E. Marczewski, Sur les congruences et les propriétés positives d'algèbres absiraites, Colloq. Math. 2 (1951), pp. 220-228.

[4] J. Mycielski, Some compactifications of general algebras, Colloq. Math. 13 (1964) pp. 1-9.

[5] W. Taylor, Atomic compactness and graph theory, Fund. Math. 65 (1969) pp. 139-145.

[6] R. B.Warfield, Purity and algebraic compactness for modules, Pacific J. Math. 28 (1969), pp. 699-719.

[7] B. Węglorz, Equationally compact algebras (I), Fund. Math. 59 (1966), pp. 289-298. 